\title{
NIPPLE REATTACHMENT BY DISLODGED POUCH YOUNG OF THE QUOKKA, SETONIX BRACHYURUS
}

\author{
R. ASHMAN,* J. SHIELD AND H. WARING \\ Department of Zoology, The University of Western Australia, \\ Nedlands 6009, Australia
}

(Received 19th Fuly 1974)

\begin{abstract}
Summary. Contrary to popular belief, marsupial pouch young will readily reattach to the nipple after detachment. Subjection of the young to quite severe surgery does not appear to prejudice the success of reattachment.
\end{abstract}

Marsupial pouch young offer considerable advantages for the study of the ontogeny of physiological systems because they are relatively undeveloped at birth compared to eutherian mammals. Full advantage has not, however, been taken of this experimental system because of the fallacy perpetuated in the early scientific literature (Wood Jones, 1923; McCrady, 1938; Troughton, 1943; Moore, 1947), and unquestioned since (e.g. Solomon, 1971), that removal from the teat causes damage to the sealed lips of the pouch young and precludes reattachment.

A West Australian marsupial, the quokka (Setonix brachyurus), has been used as an experimental animal in our laboratories for over 20 years, and considerable data have been gathered on the survival of the pouch young after experimental manipulation, and after accidental dislodgement from the teat. The pouch of the quokka is relatively elastic and the inner epithelium bearing the nipple and mammary gland can be drawn a considerable distance towards the pouch opening. This has minimized accidental detachment during experimental manipulations. The proportion of pouch young accidentally detached from the nipple during the experiments was 21 out of $300(7 \%)$ and 60 out of $230(26 \%)$ pouch young aged 1 to 10 days and 10 to 100 days, respectively.

When pouch young 10 days or older were dislodged from the teat, they were returned to the pouch and the mothers were either released immediately or held overnight in hessian sacks and released the following morning. The survival of the detached pouch young of mothers rested before release was markedly superior to that of mothers released immediately (Table 1). In the majority of cases of spontaneous reattachment, pouch young reattached to the nipple within $4 \mathrm{hr}$. The longest recorded period off the nipple when the pouch young subsequently survived was $36 \mathrm{hr}$ for a 65-day-old pouch young, although animals at about this age may detach from the nipple, and reattach at will. Even pouch young less than 5 days of age have been observed to return spontaneously to the

\footnotetext{
* Present address: Lobund Laboratory, University of Notre Dame, Notre Dame, Indiana 46556,
} U.S.A. 
teat upon return to the pouch when the mothers were allowed a period of tranquillity before release.

There is evidence that quite severe surgery can be performed on the pouch young without prejudicing spontaneous reattachment, but this conclusion cannot be statistically validated. In operations involving major surgery of the pouch young (e.g. opening the chest cavity), however, it was judged expedient to attempt to reattach the young before release of the mother. Hence, when experimental procedures on 1- to 10-day-old pouch young, accidentally detached from the teat, had been completed, the teat was reinserted in the mouth using a pair of fine curved forceps. Of twenty-two pouch young reattached by this method, nineteen survived $(87 \%)$. In no case was there

Table 1. Spontaneous reattachment of pouch young of mothers rested before release compared to mothers released immediately

\begin{tabular}{l|c|c|c}
\hline & $\begin{array}{c}\text { No. } \\
\text { detached }\end{array}$ & $\begin{array}{c}\text { No. } \\
\text { reattached }\end{array}$ & $\begin{array}{c}\% \\
\text { Reattached }\end{array}$ \\
\hline $\begin{array}{l}\text { Mothers rested } \\
\text { before release }\end{array}$ & 48 & 43 & 90 \\
$\begin{array}{c}\text { Mothers released } \\
\text { immediately }\end{array}$ & 9 & 4 & 44 \\
\hline
\end{tabular}

Table 2. Mortality after experimental manipulation of quokka pouch young

\begin{tabular}{l|c|c}
\hline Age of pouch young & $\%$ Mortality & No. of animals \\
\hline 1 to 10 days & 22 & 300 \\
10 to 100 days & 16 & 99 \\
\hline
\end{tabular}

evidence of damage to the mouth or lips of the young when using this method. Although it is desirable to perform surgery and other experimental manipulations. with the young attached to the teat, it is apparent that a high survival rate can be attained by simply reinserting the teat into the mouth of the pouch young after surgery.

The survival of the pouch young that reattached to the teat after accidental dislodgement did not differ from that of young that remained attached. The overall mortality from all sources (e.g. handling, bleeding and including failure to reattach), but excluding deaths on the table during surgery, is summarized in Table 2.

Thus, although the pouch young of the quokka appear to be better suited to experimental manipulation than those of the opossum, we suggest that, by utilizing the technique of forced reattachment, quite severe experimental manipulation may be performed on any marsupial with a high expectation of survival. 
We thank Miss S. M. Hodgson and Mr J. J. McMath, Mr J. C. Sampson and Mr R. M. Holmes for technical assistance. Dr M. M. S. Yadav's expertise in catching animals is gratefully acknowledged. Animal facilities were provided by a grant from G.S.I.R.O. to Professor H. Waring.

\section{REFERENCES}

MaCrady, E., Jr (1938) The embryology of the opossum. Am. anat. Mem. 16, 1-226.

Moore, C. R. (1947) Embryonic Sex Hormones and Sexual Differentiation. Charles C. Thomas, Springfield. Solomon, J. B. (1971) Foetal and Neonatal Immunology. North Holland, Amsterdam and London.

Troughton, E. (1943) Furred Animals of Australia. Angus \& Robertson, Sydney.

Wood Jones, F. (1923) The Mammals of South Australia. I. The Monotremes and Carnivorous Marsupials. Handbooks of the Flora and Fauna of South Australia. Government Printer, Adelaide. 